\title{
On the water-induced critical double point in a polymer solution: the role of isotopic substitution
}

\author{
B M JAFFAR ALI, S VENKATACHALAM* and A KUMAR ${ }^{\dagger}$ \\ Department of Physics, Indian Institute of Science, Bangalore 560012, India \\ * Present address: Department of Physics, University of California, San Diego, La Jolla, CA 92093 , \\ USA \\ $\dagger$ Corresponding author. \\ E-mail phyanil@physics.iisc.ernet.in
}

MS received 14 July 1997; revised 22 October 1997

\begin{abstract}
This paper examines the effect of substitution of water by heavy water in a polymer solution of polystyrene (molecular weight $=13000$ ) and acetone. A critical double point $(\mathrm{CDP})$, at which the upper and the lower partially-miscible regions merge, occurs at nearly the same coordinates as for the system [polystyrene + acetone + water]. The shape of the critical line for [polystyrene + acetone + heavy water] is highly asymmetric. An explanation for the occurrence of the water-induced CDP in [polystyrene + acetone] is advanced in terms of the interplay between contact energy dissimilarity and free-volume disparity of the polymer and the solvent. The question of the possible existence of a one-phase hole in an hourglass phase diagram is addressed in [polystyrene + acetone + water]. Our data exclude such a possibility.
\end{abstract}

Keywords. Liquid-liquid transitions; critical double point; polymer demixing; isotopic substitution.

\section{PACS Nos $64.70 ; 64.60$}

\section{Introduction}

Polymer solutions exhibit a few novel thermodynamic states, e.g., theta point, critical double point (CDP) [1,2] and tricritical point [3] induced by variations in the molecular weight of the polymer or the applied pressure. Polymer solutions are known to share the Ising universality class with binary liquid mixtures [1,2]. Polymer solutions show a high temperature partially-miscible region that terminates at lower critical solution temperature, $T_{\mathrm{L}}$ and a low temperature partially-miscible region that terminates at upper critical solution temperature, $T_{\mathrm{U}}$ [4-6]. The CDP is defined as a point where $T_{\mathrm{L}}$ and $T_{\mathrm{U}}$ are made to merge, i.e., the miscibility gap $\Delta T\left[=\left|T_{\mathrm{L}}-T_{\mathrm{U}}\right|\right]$ becomes zero. Other examples of systems that display CDP are aqueous alcohols [secondary butyl alcohol + tertiary butyl alcohol + water] [7], binary gases (Ne-Kr) [8] and mixtures of liquid sulphur and hydrocarbons (e.g., benzene or toluene). A double critical point, DCP (where a closed-loop phase diagram vanishes) is identical to a CDP except for some finer details concerning the approach to these points [6].

In practice, the role of pressure in multicomponent liquid systems as well as polymer solutions is simulated by isotopic/isomeric substitutions $[6,7,9]$ or by additional 
chemical components $[1,6]$. It was first reported [1] from this laboratory that minute quantities of water can induce a CDP in a solution of [polystyrene (molecular weight $13000)+$ acetone]. This procedure had yielded [1] an unusually close approach to CDP in [polystyrene(PS) + acetone $(\mathrm{AC})+$ water].

The high-temperature immiscibility of (polymer plus poor solvent) systems can be understood within the framework of the Prigogine solution theory as modified by Patterson $[4,5,10]$. The quantity of interest here is the interaction parameter, $\chi$, which reflects the interplay of two competing terms, viz., the contact energy dissimilarity and free-volume disparity of the polymer and the solvent. The expression for $\chi$, as given by the Patterson and the Flory theories [4], is

$$
\chi=\frac{-U_{1}}{R T} \nu^{2}+\left(\frac{C_{p 1}}{2 R}\right) \tau^{2}
$$

where $U_{1}$ is the configurational energy of the solvent, $C_{p 1}$, the first derivative of $U_{1}$ with respect to $T$, is the configurational heat capacity of the solvent, and $R$ is the gas constant. The parameter $\nu^{2}$ expresses the difference of the cohesive energy and size between the solvent molecule and the polymer segment. The parameter $\tau$ reflects the free-volume change, which occurs due to large differences in the thermal expansion coefficients of the polymer and the solvent.

The extent of miscibility or the extent of stability of the mixed state is determined by the Flory theory from the combinatorial part of $\Delta G_{M}$, viz., the total free energy of mixing. From this we get an expression for the critical value of $\chi, \chi_{\mathrm{c}}$, as follows $[4,10]$

$$
\chi_{c}(T, P)=\frac{1}{2}\left[1+\left(\frac{v_{1}}{v_{2}}\right)^{1 / 2}\right]^{2},
$$

where $v_{1}$ and $v_{2}$ are the molar volumes of the solvent and the polymer, respectively. It should be noted that $\chi_{c}$ decreases with an increase in molecular weight of the polymer.

The first term in (1) usually decreases with temperature $(T)$, whereas the second term is an increasing function of temperature. Typically, a $\chi-T$ curve (figure 1) has a minimum

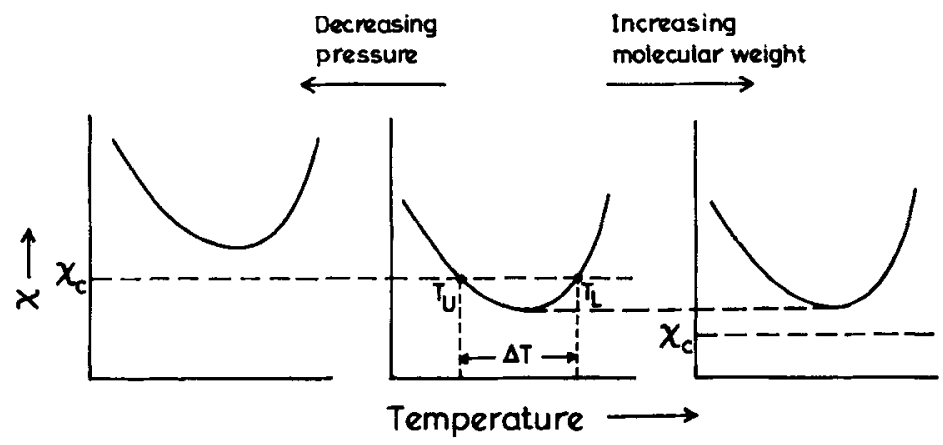

Figure 1. The behaviour of the $\chi-T$ curve, and $\chi_{\mathrm{c}}$ with change in pressure and molecular weight of the polymer. These changes lead to an alteration in the miscibility behaviour (i.e., $\Delta T$ value) and the realization of a critical double point $(\Delta T=0)$ as well as appearance of an hourglass phase diagram. 
in $\chi$ (i.e., $\partial \chi / \partial T \rightarrow 0$ or $\partial T / \partial \chi \rightarrow \infty$ ). A finite $\Delta T$ is attained when the line corresponding to $\chi_{\mathrm{c}}$ intersects this curve at temperatures $T_{\mathrm{U}}$ and $T_{\mathrm{L}}$. The difference between these two temperatures, $\Delta T\left[=\left|T_{\mathrm{L}}-T_{\mathrm{U}}\right|\right]$, denotes the size of the miscibility gap.

Increasing the molecular weight of the polymer without changing the nature of the $\chi-T$ curve leads to a decrease in the $\chi_{c}$ value (figure 1). Hence, at some value of molecular weight, the $\chi_{\mathrm{c}}$ line can become tangential to the $\chi-T$ curve, leading to the occurrence of a $\operatorname{CDP}(\Delta T=0)$. The situation in which the $\chi-T$ curve lies above the $\chi_{\mathrm{c}}$ value corresponds to the hourglass phase diagram.

Alternatively, a decrease in pressure $(P)$ can increase the free-volume differences between the two components and thus shift the $\chi-T$ curve upwards (figure 1). However, the $\chi_{\mathrm{c}}$ line does not vary with pressure. Thus a condition of $\chi_{\mathrm{c}}$ line being tangential to the $\chi-T$ curve is again realized, resulting in a CDP. Any further decrease in pressure yields an hourglass phase diagram. In this manner, one can explain the occurrence of a CDP in a polymer solution in two different ways.

Changing the molecular weight of polystyrene from 4800 to 19800 in [PS $+\mathrm{AC}$ ] leads to a CDP at a molecular weight of 19800 and a temperature of $\simeq 70^{\circ} \mathrm{C}$ [4]. By variation of pressure in [PS (molecular weight 20000) $+\mathrm{AC}$ ], the CDP is realized [2,5] at a pressure of 3.33 bar and a temperature of $69.86^{\circ} \mathrm{C}$. We have shown earlier [1] that a small quantity of the third component, i.e., water, can induce a CDP in [PS (molecular weight $13000)+\mathrm{AC}]$ at $89.258^{\circ} \mathrm{C}$ for 0.007 weight fraction of water.

The objectives of this work are: (a) to discern the effect of isotopic (H/D) substitution on the nature of CDP in [PS $+\mathrm{AC}$ ] solutions, i.e., to impart generality to our earlier observation of CDP in this system, and (b) to uncover finer details of hourglass phase diagram in close neighbourhood of CDP - in the light of the observation [11] of a onephase hole or loop inside the hourglass configuration.

\section{Experiment}

Samples were made using polystyrene (PS) (Pressure Chemical Co.) of molecular weight 13000 and polydispersity of less than $6 \%$, i.e., $M_{w} / M_{n}=1.06$, where $M_{w}$ is weight average molecular weight and $M_{n}$ is number average molecular weight. The acetone (AC) was of spectroscopic grade $(99.8 \%$, Ranbaxy, India). Heavy water was of isotopic purity 99.6\% (BARC, India). Water used was triple distilled in an all-quartz distiller.

The miscibility range, $\Delta T\left(=T_{\mathrm{L}}-T_{\mathrm{U}}\right)$, is controlled by the weight fraction $(W)$ of water or heavy water in the polymer solution [PS $+\mathrm{AC}]$. We believe that $W$ simulates the condition of a thermodynamic field in the sense that the nature of $\mathrm{W}-\mathrm{T}$ graph is similar to that of a P-T plot for this system [2]. The amount of water/heavy water involved is extremely small, varying from 0.0008 to 0.0080 weight fraction of the total mixture. To permit the addition of such small quantities, a stringent sample preparation procedure was adopted.

The critical concentration ( $w_{\mathrm{c}}$, weight fraction of polystyrene) for [PS $+\mathrm{AC}+$ heavy water] system was determined and it was found to be nearly constant at 0.23 for all $\Delta T \mathrm{~s}$, i.e., $w_{\mathrm{c}}$ had no $\Delta T$ dependence. So, to realize the critical line correctly, it was necessary to maintain the constancy of the critical concentration $\left(w_{\mathrm{c}}\right)$ for samples with varying $\Delta T \mathrm{~s}$. Stock solutions of $[\mathrm{AC}+$ heavy water] were prepared in the required concentrations 


\section{B M Jaffar Ali, S Venkatachalam and A Kumar}

corresponding to different values of $W$ and concomitant with a fixed amount of PS ( $w_{\mathrm{c}}$ ) under similar conditions. Requisite amounts of this solution were added to the correctly weighed amounts of PS. A stock solution of [AC + heavy water] was made so as to overcome the problem of actual addition of extremely small quantities of heavy water $(\simeq 0.0005 \mathrm{~g})$ to the $[\mathrm{PS}+\mathrm{AC}]$ system.

In the case of the determination of hourglass curve in [PS + AC + water] system, the quantity $W$ (weight fraction of water in the mixture) is to be kept constant. A stock solution of [AC + water] was prepared. However, the quantities of $\mathrm{AC}$ and water added were in such proportions that they corresponded to a weight fraction $(w)$ of polystyrene $=0.5$. A sample with $w=0.5$ was prepared from this stock solution. Subsequently, several samples with different weight fractions of polystyrene $(w<0.5)$ were prepared for a fixed $W$. The total amount of water and acetone required for each of these samples was calculated. An amount of stock solution containing the required amount of water was then added. The requisite amount of additional acetone was calculated and added separately since the stock solution of [AC + water] was prepared for $w=0.5$. Once again, the difficulty of having to add exceptionally small quantities of water was circumvented by using the stock solution of $[\mathrm{AC}+$ water]. This method

Table 1. Details of the samples for [polystyrene + acetone + heavy water] used to study the shape of the critical line (figures 2-4). $W$ denotes the weight fraction of heavy water and $w_{c}$ signifies the critical weight fraction of polystyrene. $T_{\mathrm{U}}$ and $T_{\mathrm{L}}$ indicate the upper and lower consolute points, respectively.

\begin{tabular}{lcccc}
\hline$W$ & $w_{\mathrm{c}}$ & $T_{\mathrm{U}}\left({ }^{\circ} \mathrm{C}\right)$ & $T_{L}\left({ }^{\circ} \mathrm{C}\right)$ & $\begin{array}{c}\Delta T\left({ }^{\circ} \mathrm{C}\right) \\
\left(T_{\mathrm{L}}-T_{\mathrm{U}}\right)\end{array}$ \\
\pm 0.0001 & \pm 0.003 & \pm 0.03 & & 85.7 \\
0.0020 & 0.23 & 33.10 & 118.8 & \pm 1.03 \\
& & & \pm 1 & 78.27 \\
0.0030 & 0.23 & 38.730 & 117.0 & \pm 1.03 \\
& & & \pm 1 & 68.736 \\
0.0040 & 0.23 & 46.264 & 115.0 & \pm 1.03 \\
& & & \pm 1 & 58.75 \\
0.0050 & 0.23 & 53.75 & 112.5 & \pm 0.43 \\
& & & \pm 0.4 & 47.153 \\
0.0060 & 0.23 & 62.247 & 109.4 & \pm 0.13 \\
& & & \pm 0.1 & 40.718 \\
0.0065 & 0.23 & 66.782 & 107.5 & \pm 0.13 \\
& & & \pm 0.1 & 33.776 \\
0.0070 & 0.23 & 70.824 & 104.6 & \pm 0.13 \\
& & & \pm 0.1 & 24.285 \\
0.0075 & 0.23 & 77.315 & 101.6 & \pm 0.13 \\
& & & \pm 0.1 & 14.798 \\
0.0078 & 0.23 & 83.802 & 98.6 & \pm 0.08 \\
& & & \pm 0.05 & 7.045 \\
0.0080 & 0.23 & 88.68 & 95.725 & \pm 0.08 \\
& & & 94.115 & 3.45 \\
0.00805 & 0.23 & 90.665 & \pm 0.05 & \pm 0.08 \\
\hline & & & & \\
\hline
\end{tabular}


Table 2. The coexistence data for the hourglass coexistence curve shown in figure $6 . w$ denotes the weight fraction of polystyrene.

\begin{tabular}{|c|c|c|c|}
\hline$T\left({ }^{\circ} \mathrm{C}\right)$ & $\begin{array}{c}w^{+} \\
\pm 0.003\end{array}$ & $\begin{array}{c}w^{-} \\
\pm 0.003\end{array}$ & $\begin{array}{c}\Delta w \\
\left(w^{+}-w^{-}\right)\end{array}$ \\
\hline \multicolumn{4}{|c|}{$W=0.0072$} \\
\hline $\begin{array}{r}103.5 \\
\pm 0.1\end{array}$ & 0.45 & 0.158 & 0.292 \\
\hline $\begin{array}{r}101.2 \\
\pm 0.1\end{array}$ & 0.436 & 0.17 & 0.266 \\
\hline $\begin{array}{l}97.523 \\
\pm 0.05\end{array}$ & 0.40 & 0.19 & 0.210 \\
\hline $\begin{array}{l}94.710 \\
\pm 0.05\end{array}$ & 0.36 & 0.197 & 0.163 \\
\hline $\begin{array}{l}94.202 \\
\pm 0.05\end{array}$ & 0.352 & 0.20 & 0.152 \\
\hline $\begin{array}{l}92.730 \\
\pm 0.03\end{array}$ & 0.34 & 0.204 & 0.136 \\
\hline $\begin{array}{l}91.421 \\
\pm 0.03\end{array}$ & 0.31 & 0.206 & 0.104 \\
\hline $\begin{array}{l}90.242 \\
\pm 0.03\end{array}$ & 0.281 & 0.21 & 0.071 \\
\hline $\begin{array}{l}88.420 \\
\pm 0.03\end{array}$ & 0.302 & 0.21 & 0.092 \\
\hline $\begin{array}{r}87.82 \\
\pm 0.03\end{array}$ & 0.31 & 0.209 & 0.101 \\
\hline $\begin{array}{l}85.465 \\
\pm 0.03\end{array}$ & 0.34 & 0.208 & 0.132 \\
\hline $\begin{array}{r}83.76 \\
\pm 0.03\end{array}$ & 0.36 & 0.204 & 0.156 \\
\hline $\begin{array}{l}83.424 \\
\pm 0.03\end{array}$ & 0.20 & 0.36 & 0.160 \\
\hline $\begin{array}{l}80.545 \\
\pm 0.03\end{array}$ & 0.40 & 0.19 & 0.210 \\
\hline $\begin{array}{l}77.220 \\
\pm 0.03\end{array}$ & 0.45 & 0.173 & 0.277 \\
\hline $\begin{array}{l}76.835 \\
\pm 0.03\end{array}$ & 0.454 & 0.17 & 0.284 \\
\hline $\begin{array}{l}70.865 \\
\pm 0.03\end{array}$ & $\cdots$ & 0.14 & \\
\hline
\end{tabular}

allowed for a complete control over $W$ and $w$ as well as a constancy of conditions under which different samples were prepared. A typical sample (in all preparations) was about $0.15-0.20 \mathrm{ml}$ in volume. This typically contained about $0.0005 \mathrm{~g}$ of water.

Samples were prepared in pyrex cells with Teflon high vacuum stopcocks (Rotaflo). A serious problem encountered in generating the coexistence curve and the critical line is the drift of $\Delta T$, particularly for its small values. This difficulty stems from the acute steepness of the coexistence surface in the vicinity of the CDP $[1,2,6-8]$. Neither 
degassing to remove trapped atmospheric oxygen nor preparation of the sample under dry nitrogen improved the temporal stability of the samples for any $\Delta T$. Because only very small quantities of water or heavy water are involved in diminishing the $\Delta T$ (tables 1 and 2), a great deal of caution has to be exercised in maintaining the constancy of $W$. Reliable data could be obtained only if phase separation temperatures were determined soon after the samples were made.

Phase separation temperatures were determined visually $[1,6]$ by monitoring the onset of intense opalescence. Criticality of the samples was ascertained by the equal-volume criterion. For off-critical samples, phase separation temperatures were marked by the actual appearance of meniscus. The visual measurements were done in a well-stirred paraffin thermostat with a temperature stability better than $\pm 1 \mathrm{mK}$ in the temperature range $35-95^{\circ} \mathrm{C}$. The temperature is measured to an absolute accuracy of $\pm 60 \mathrm{mK}$ with a platinum resistance thermometer in conjunction with a resistance bridge (Model F17, ASL, UK). The determination of phase separation temperature in the range $95-110^{\circ} \mathrm{C}$ was carried out in a polyethylene glycol thermostat with a temperature stability of $\pm 50 \mathrm{mK}$. For temperature above $110^{\circ} \mathrm{C}$, a polyethylene glycol thermostat with a temperature stability of $\pm 0.5 \mathrm{~K}$ was used. It was essential to stir the sample during the course of detection of phase separation temperature. A typical run to determine the upper critical solution temperature $\left(T_{\mathrm{U}}\right)$ lasted 6-8 hours and the temperature range scanned was $2^{\circ} \mathrm{C}$. Determination of lower critical solution temperature $\left(T_{\mathrm{L}}\right)$ lasted 4 hours and the temperature range scanned was about $2^{\circ} \mathrm{C}$.

The line of critical points was determined in a single set of experiments. It was observed that the temperature range of persistence of critical opalescence is smaller for [PS $+\mathrm{AC}+$ heavy water] than for [PS $+\mathrm{AC}+$ water]. Table 1 gives the details of the determination of critical line for [PS $+\mathrm{AC}+$ heavy water] and table 2 provides the data for the hourglass coexistence curve for [PS $+\mathrm{AC}+$ water].

\section{Results}

The data for $T_{\mathrm{U}}$ and $T_{\mathrm{L}}$ [(PS $+\mathrm{AC}+$ heavy water), table 1] were fitted to the following expression

$$
\left(T-T_{\mathrm{CDP}}\right)=A_{1}\left(W-W_{\mathrm{CDP}}\right)^{A_{2}}
$$

where $T_{\mathrm{CDP}}$ and $W_{\mathrm{CDP}}$ are the coordinates of the CDP. The data were analysed using a modified form of the program CURFIT [1]. The fit was deemed to be good if the minimum of reduced $\chi^{2}\left(\chi_{\nu}^{2}\right)$ was close to 1 and resulting residuals had random distribution. The best-fit values of the parameters are

$$
A_{1}=321.02 \pm 4.59 ; \quad A_{2}=0.6046 \pm 0.0080 ; \quad \chi_{\nu}^{2}=1.213
$$

and

$$
A_{1}^{\prime}=93.40 \pm 0.54 ; \quad A_{2}^{\prime}=0.4380 \pm 0.0018 ; \quad \chi_{\nu}^{2}=2.220
$$

where $A_{1}$ and $A_{2}$ refer to the lower section of the critical curve, and $A_{1}^{\prime}$ and $A_{2}^{\prime}$ pertain to the upper section of the critical curve. The coordinates of CDP are given by $T_{\mathrm{CDP}}=92.275 \pm 0.035^{\circ} \mathrm{C}$ and $W_{\mathrm{CDP}}=0.00806 \pm 0.00010$. The solid line in figure 2 is 
Critical double point in a polymer solution

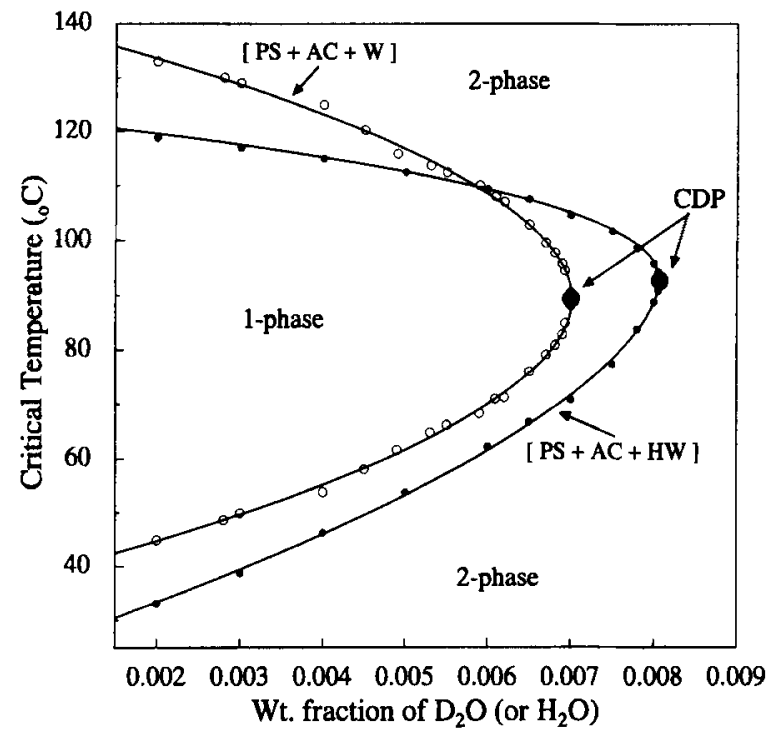

Figure 2. A comparative portrayal of the shape of the critical line for [polystyrene + acetone + water] and for [polystyrene + acetone + heavy water] in terms of weight fraction of water or heavy water. Extrema of the curves denote the critical double point (CDP).

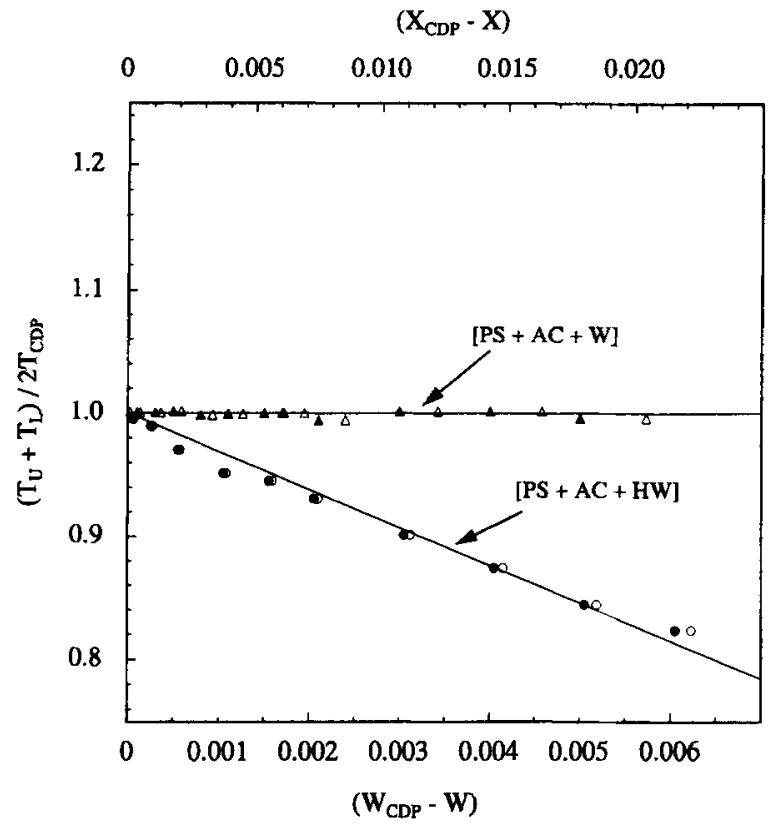

Figure 3. Illustration of the acute asymmetry in the line of critical points for [polystyrene + acetone + heavy water], in contrast to the case for [polystyrene + acetone + water]. The filled symbols refer to the weight fractions $(W)$ of water/heavy water, whereas the corresponding open symbols relate to the mole fraction $(X)$ of water or heavy water. 


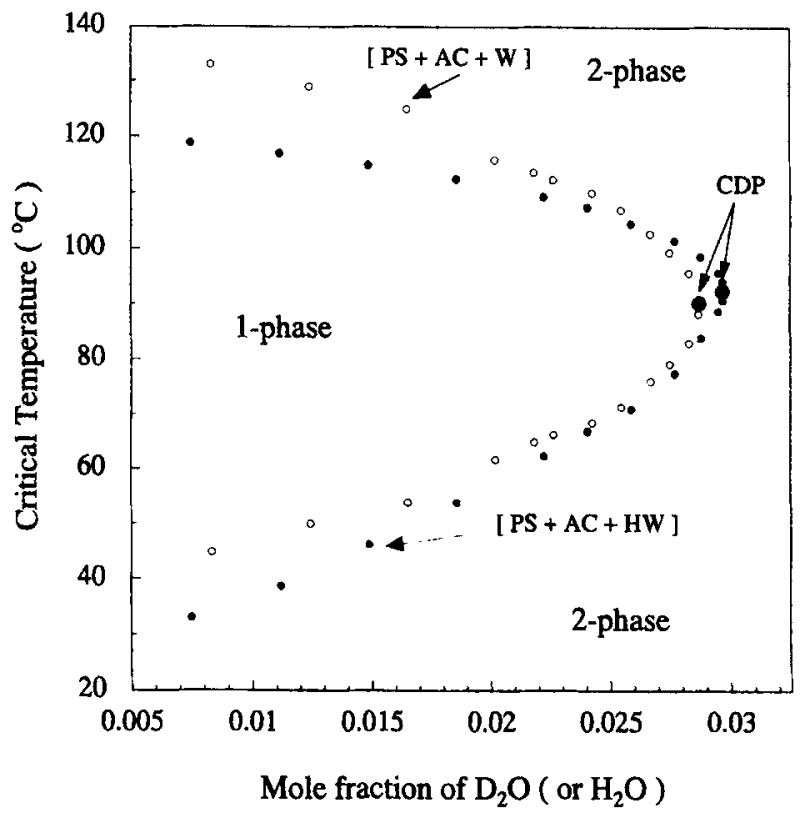

Figure 4. A comparative portrayal of the shape of the critical line for [polystyrene + acetone + water] and for [polystyrene + acetone + heavy water] in terms of mole fraction of water or heavy water. Extrema of the curves denote the critical double point (CDP). Note the much closer values of $X_{\mathrm{CDP}}$ for the two curves in comparison to the situation depicted in figure 2 .

generated by (3). This curve is found to be grossly asymmetric, as reflected in figure 3 . If data reduction is confined to the close proximity of CDP, the critical line is fairly symmetric (figure 3). In comparison, the line of critical point for [PS $+\mathrm{AC}+$ water] was found to be both symmetric and parabolic [1]. The coordinates of the CDP for [PS + AC + water] are: $T_{\mathrm{CDP}}=89.258 \pm 0.03^{\circ} \mathrm{C}$ and $W_{\mathrm{CDP}}=0.00702 \pm 0.00010$.

In view of the fact that molar volume of water and heavy water are almost identical, the nature of critical lines for [PS + AC + water] and [PS + AC + heavy water] was also examined using mole fractions $(X)$ of water or heavy water. Figure 4 reveals that the two CDPs are much closer in terms of mole fractions, though the asymmetry of the critical line still persists (figures 2 and 3). For instance, the weight fraction ratio of CDPs is 1.15 (figure 2) and the mole fraction ratio of CDPs is virtually unity (1.03), as evidenced by figure 4 .

The reentrant critical line in ternary mixture [7] of [secondary butylalcohol + tertiary butylalcohol + water] and in binary gases [8], e.g., ( $\mathrm{Ne}-\mathrm{Kr}$ ) are quite asymmetric, particularly away from a CDP.

Although polystyrene (PS) used in the experiments involving [PS $+\mathrm{AC}+$ heavy water] was of the same stated purity and polydispersity as the one used [1] in [PS + AC + water], it belonged to a different lot. This fact may have led to the differences in the limiting critical temperatures of the two curves shown in figure 2 for the binary limit of $[\mathrm{PS}+\mathrm{AC}]$. However, the $\Delta T$ values for [PS $+\mathrm{AC}]$ seem to be reasonably closer for the two curves in figure 2. 


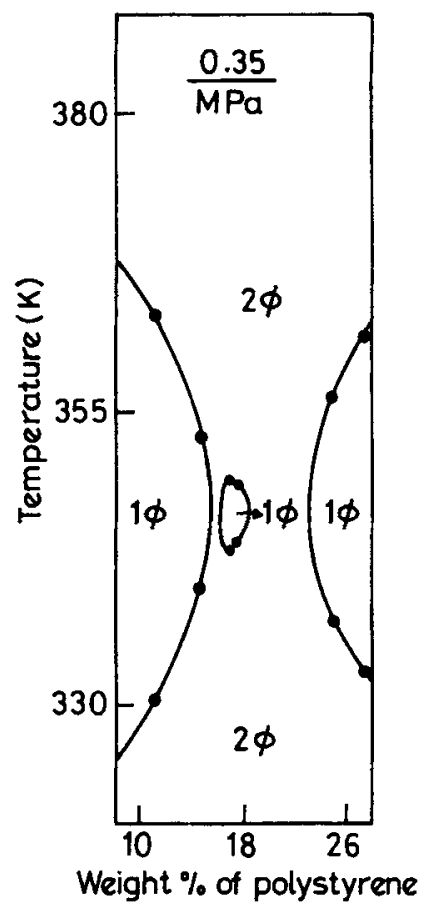

Figure 5. Hourglass coexistence curve for [polystyrene (molecular weight 22400) + acetone] system at $0.35 \mathrm{MPa}(3.5 \mathrm{bar})$. Note the unusual existence of one-phase $(1 \phi)$ hole inside the two-phase region (after ref. [11]).

The closest approach to CDP in [PS + AC + heavy water] is signified by $\Delta T=3.45^{\circ} \mathrm{C}$ (table 1, figure 2). In ternary liquid mixtures, the critical point does not coincide with the apex or the extremum ( $T_{\mathrm{EL}}$ or $\left.T_{\mathrm{EU}}\right)$ of the relevant coexistence curve $[1,6]$. Therefore $\Delta T=3.45^{\circ} \mathrm{C}$ actually corresponds to the approach to an effective CDP to within a few hundreds of $\mathrm{mK}$ [1]. For instance, for [PS $+\mathrm{AC}+$ water], $\Delta T=2.34^{\circ} \mathrm{C}$ related to $\Delta T_{E}$ $\left(=T_{\mathrm{EL}}-T_{\mathrm{EU}}\right)=194 \mathrm{mK}[1]$.

In a bid to locate the existence of a one-phase $(1 \phi)$ hole [11] in an hourglass phase diagram (figure 5), we explored the relevant phase boundary for [PS + AC + water]. This hourglass phase diagram (figure 6) was determined for $W(=0.0072)>W_{\mathrm{CDP}}$ $(=0.00702)$ so that it met the condition of being in the close proximity of CDP. As figure 6 illustrates, the entire two-phase $(2 \phi)$ region of the hourglass phase diagram is devoid of any one-phase hole.

\section{Discussion}

We had earlier demonstrated [1] that the introduction of very small quantities of water to a polymer solution of [PS $+\mathrm{AC}$ ] can yield a CDP. The same finding has been reinforced by the substitution of water by heavy water in [PS $+\mathrm{AC}]$. There does not seem to be any profound difference between the CDP in [PS + AC + water] and that in [PS + AC + heavy water] (figure 4). This feature may possibly be due to the fact that minute quantities 


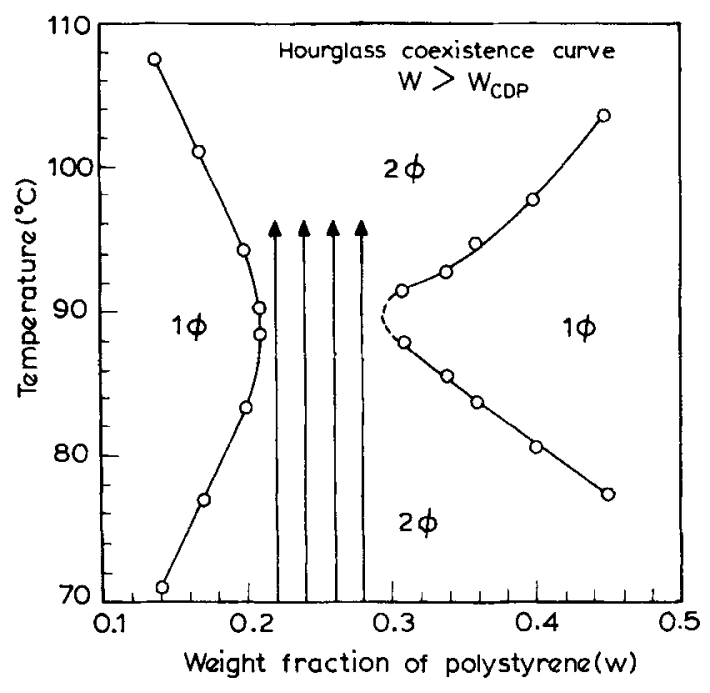

Figure 6. Hourglass coexistence curve for [polystyrene (molecular weight 13000) + acetone + water] for $W(=0.0072)>W_{\mathrm{CDP}}(0.00702)$, where $W$ is the weight fraction of water in the mixture. Arrows indicate the region scanned to uncover the possible existence of a one-phase $(1 \phi)$ hole in the vicinity of critical double point, CDP $\left(w_{\mathrm{CDP}}=0.23, T_{\mathrm{CDP}}=89.258^{\circ} \mathrm{C}\right)$.

of either water or heavy water are adequate to realize the merger of upper and lower partially-miscible regions. The earlier experiments concerning isotopic substitution in polymer solution systems had concluded that the deuteration of polymer simulated the applied pressure and the deuteration of the solvent led to simulation of a negative pressure [9].

A striking outcome of $\mathrm{H} / \mathrm{D}$ substitution in [PS $+\mathrm{AC}+$ water] is the asymmetry in the critical curve (figures 2 and 3 ). It is stressed that in the close proximity of the CDP, both the curves are reasonably symmetric (figure 3 ). For example, the asymmetry is no more than $\sim 5 \%$ for $\left(W_{\mathrm{CDP}}-W\right) \leq 0.001$. This composition range implies a $\Delta T \leq 24^{\circ} \mathrm{C}$. From the standpoint of the evolution of critical behaviour near $\mathrm{CDP}$, the region of $\Delta T \leq 24^{\circ} \mathrm{C}$ is quite adequate [6]. Besides, Landau-Ginzburg theory and lattice-solution models demand a symmetric, parabolic critical line only in the immediate vicinity of a DCP or a CDP - at least for systems involving small molecules [6]. A possible explanation of the asymmetry of the critical curve for [PS $+\mathrm{AC}+$ heavy water], in contrast to that for [PS $+\mathrm{AC}+$ water], may be related to the slight differences in the nature of the exact $\chi-T$ curves for the two cases. Beyond these considerations, we have no other explanation to account for the asymmetry of the critical curve of [PS $+\mathrm{AC}+$ heavy water] in the region away from the CDP.

The role of water or heavy water in suppressing $\Delta T$ in [PS $+\mathrm{AC}$ ] has to be probed quantitatively. Qualitatively, one can attempt to understand it in terms of the behaviour of $\chi$ within the framework of the Patterson-Delmas theory $[4,5,10]$. Both water and heavy water constitute a very bad solvent for polystyrene. Its addition to the system can be viewed as acting to lower the solvent quality of acetone. This would imply an increase in the contact energy dissimilarity between the polymer and the solvent and, hence, an 


\section{Critical double point in a polymer solution}

increase in the contribution due to the first term in $\chi$ (in eq. (1)) upon the addition of water or heavy water to the system. The contribution to the second term in $\chi$ (eq. (1)) stems from the free-volume disparity (due to the difference in the degree of thermal expansion) between the polymer and solvent molecules. The coefficient of thermal expansion $(\alpha)$ of water and heavy water is much lower than that of acetone $\left(\alpha_{W}=0.257 \times 10^{-3} \mathrm{deg}^{-1}\right.$, $\alpha_{\mathrm{HW}}=0.185 \times 10^{-3} \mathrm{deg}^{-1}$ and $\alpha_{\mathrm{AC}}=1.42 \times 10^{-3} \mathrm{deg}^{-1}$ ). For PS, as expected, $\alpha$ has a much lower value than that of $\mathrm{AC}$, water and heavy water $\left(\alpha_{\mathrm{PS}}=0.0824 \times 10^{-3} \mathrm{deg}^{-1}\right)$. The addition of water or heavy water should, thus, lead to a reduction in the contribution to $\chi$ originating from the free-volume disparity (second term in eq. (1)). The overall behaviour of $\chi$ is a consequence of the competition between these two terms (eq. (1)). $\chi_{\mathrm{c}}$ depends only on the ratio of the molar volume of the polymer to the molar volume of the solvent (i.e., on the ratio of the two molecular weights). The addition of water or heavy water does not alter this ratio and, hence, one does not expect $\chi_{\mathrm{c}}$ to change drastically. The addition of water/heavy water leads to a net increase in $\chi$ (as $\chi_{\mathrm{c}}$ remains constant), which, in turn, brings about a reduction in $\Delta T$. It can be argued that the desired net increase in $\chi$ is possible only if the increase in $\chi$ due to the contact energy dissimilarity term far exceeds the reduction in $\chi$ brought about by the free-volume disparity term. In a gross sense, the addition of water is similar to the reduction in pressure (figure 1) in that it raises $\chi$ (leaving $\chi_{\mathrm{c}}$ constant) and thus reduces $\Delta T$, i.e., the region of miscibility. The fact that $X_{\mathrm{CDP}}$ for [PS $+\mathrm{AC}+$ heavy water] is slightly greater than $X_{\mathrm{CDP}}$ for [PS $+\mathrm{AC}+$ water] (figure 4) may be attributed to somewhat lower $\alpha$ for heavy water in comparison to that for water. However, in the absence of a clear microscopic understanding, the role of water or heavy water needs to be elucidated further.

An intriguing part of our observations is the absence of one-phase hole in hourglass configuration (figure 6). The nature of the thermodynamic state where this one-phase hole can be shrunk to a point is anticipated to be tricritical, and it reduces to a triple point in the monodisperse limit of the polymer [11]. The behaviour near such a novel thermodynamic state has not yet been experimentally ascertained. However, no one-phase hole was observed [11] for isotopically substituted [PS + AC]. Perhaps the much lower molecular weight of the polymer in our system could have masked the appearance of this unusual feature. Further experimental and analytical work in this direction is required before any firm conclusions can be reached.

It should be rewarding to compare normal and isotopically substituted [PS $+\mathrm{AC}+$ water] solutions in terms of the dynamics of phase separation, and other contexts $[6,9]$.

\section{Acknowledgements}

The authors are thankful to T Narayanan and E S R Gopal for their advice and encouragement. They benefitted immensely from the illuminating and inspiring correspondence with D Patterson, R L Scott and M Nakata. The cooperation of Jaby Jacob and T Chacko for style-editing is appreciated. One of the authors (BMJA) thanks the University Grants Commission for the award of a Research Fellowship. The pivotal financial support for this research by the Department of Atomic Energy and the Deparment of Science and Technology is gratefully acknowledged. The authors are indebted to the referee for extremely useful suggestions. 


\section{B M Jaffar Ali, S Venkatachalam and A Kumar}

\section{References}

[1] S Venkatachalam, A Kumar and E S R Gopal, J. Chem. Phys. 103, 6645 (1995)

[2] A G Casielles, R G Rubio, F Monroy and F Ortega, Phys. Rev. E49, 1404 (1994)

H Hosokawa, M Nakata and T Dobashi, J. Chem. Phys. 98, 10078 (1993)

[3] W Shen, G R Smith, C M Knobler and R L Scott, J. Phys. Chem. 94, 7943 (1990)

T Dobashi and M Nakata, J. Chem. Phys. 99, 1419 (1993)

[4] K S Siow, G Delmas and D Patterson, Macromolecules 5, 29 (1972)

[5] L Zeman and D Patterson, J. Phys. Chem. 76, 1214 (1972)

[6] T Narayanan and A Kumar, Phys. Rep. 249, 135 (1994)

[7] D L Sidebottom and C M Sorensen, J. Chem. Phys. 89, 1608 (1988)

[8] R J Tufeu, P H Keyes and W B Daniels, Phys. Rev. Lett. 35, 1004 (1975)

[9] M Luszczyk, L P Rebelo and W A Van Hook, Macromolecules 28, 745 (1995)

[10] D Patterson, Macromolecules 2, 672 (1969)

[11] L P Rebelo and W A Van Hook, J. Polym. Sci. B31, 895 (1993) 